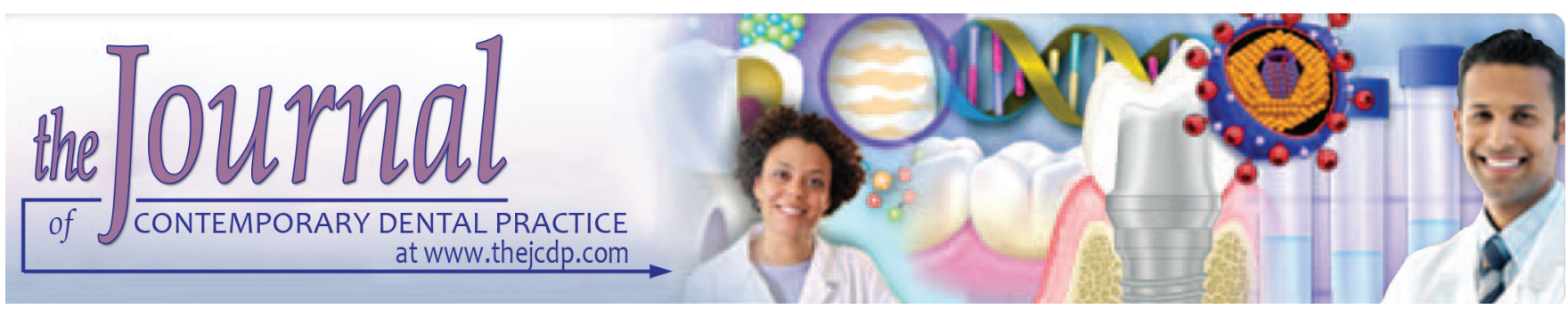

\title{
Pentraxin-3 Levels in Gingival Crevicular Fluid during Canine Retraction with Nickel-Titanium Coil Spring and Active Tieback
}

${ }^{1}$ Rakesh Thukral, ${ }^{2}$ Sukhpreet Mangat, ${ }^{3}$ Anvesha Ganguly, ${ }^{4}$ Sanket S Agarkar, ${ }^{5}$ Harleen Bali, ${ }^{6}$ Shekhar Grover

\begin{abstract}
Aim: Orthodontic treatment is routinely carried out in patients with the purpose of correcting various forms of dental malocclusions. Retraction of the canines can be achieved either individually or along with incisor. Pentraxin-3 (PTX-3) is regarded as the true independent indicator of disease activity. Hence, we undertook the present study to assess and compare the level of PTX-3 in patients undergoing canine retraction with active tieback and Nickel-Titanium (NiTi) coil spring.

Materials and methods: The present study included assessment of 25 patients that underwent canine retraction as a part of fixed orthodontic treatment. In the maxillary right and left quadrant, active tieback and NiTi coil spring were used respectively. Gingival crevicular fluid samples were collected 1 hour prior to the commencement of orthodontic canine retraction procedure followed by collection at following time intervals: 1 hour, 1,7 , and 14 days after the start of canine retraction procedure. Enzyme-linked immunosorbent assay kit was used for analysis of the samples as per manufacturer's instructions. All the data were recorded and compiled. All the results were analyzed by Statistical Package for the Social Sciences software.
\end{abstract}

Results: Nonsignificant difference was observed in the values of PTX-3 at baseline and 1 hour. While comparing the mean

\footnotetext{
${ }^{1}$ Department of Orthodontics, Modern Dental College \& Research Centre, Indore, Madhya Pradesh, India

${ }^{2}$ Private Practice, Orthodontist, Mumbai, India

${ }^{3}$ Private Practice, Orthodontist, Kolkata, India

${ }^{4}$ Department of Orthodontics, Dr. D. Y. Patil Dental College \& Hospital, Pune, Maharashtra, India

${ }^{5}$ Private Practice, Oral Medicine and Radiodiagnosis, Jammu India

${ }^{6}$ Department of Community Dentistry, Maulana Azad Dental College \& Hospital, New Delhi, India

Corresponding Author: Shekhar Grover, Department of Community Dentistry, Maulana Azad Dental College \& Hospital, New Delhi, India, Phone: +919501544877 , e-mail: drshekhagrover@gmail.com
}

PTX-3 values between different time intervals in both the study groups, significant difference was observed.

Conclusion: In comparison to the active tieback, NiTi coil exhibited faster space closure rate.

Clinical significance: In patients undergoing orthodontic treatment, PTX-3 is associated with periodontal remodeling under the effect of orthodontic forces.

Keywords: Active tieback, Nickel-Titanium, Pentraxin-3.

How to cite this article: Thukral R, Mangat S, Ganguly A, Agarkar SS, Bali H, Grover S. Pentraxin-3 Levels in Gingival Crevicular Fluid during Canine Retraction with Nickel-Titanium Coil Spring and Active Tieback. J Contemp Dent Pract 2017; 18(8):710-713.

\section{Source of Support: Nil}

Conflict of Interest: None

\section{INTRODUCTION}

Orthodontic treatment involves use of various forms of mechanical forces for the induction of bone remodeling with the purpose of correcting various forms of dental malocclusions. On the pressure side of the forces, resorption of the bone occurs, whereas on the tension side, deposition of the bone occurs. ${ }^{1}$ One of the common orthodontic treatment procedures used after the extraction of first premolars is canine retraction, which is followed by incisor retraction. Various extraoral and intraoral mechanics are employed for the conservation of anchorage in cases with maximum anchorage use. Mini-screws serve as one of the best intraoral methods for conserving the anchorage. ${ }^{2}$

Retraction of the canines can be achieved either individually or along with incisor. En masse retraction refers to the canine retraction cases done along with anterior teeth. ${ }^{3,4}$ Pentraxin-3 (PTX-3) comprises group of inflammatory mediators and acute-phase reaction markers. 
The PTX type which is regarded as the true independent indicator of disease activity is PTX-3. Periodontal literature has very limited data on the association of PTX-3 and various systemic disorders. ${ }^{5}$ Coil springs [Nickel-Titanium (NiTi)] are the common auxiliaries used for space closing in patients undergoing orthodontic treatment. Another component commonly preferred for extraction space closure is active tieback..$^{6,7}$ Hence, we undertook the present study to assess and compare the level of PTX-3 in patients undergoing canine retraction with active tieback and NiTi coil spring.

\section{MATERIALS AND METHODS}

The present study was conducted in the Department of Orthodontics of the dental institute and included assessment of 25 patients that underwent canine retraction as a part of fixed orthodontic treatment from June 2012 to July 2016. Ethical approval was taken from the institutional ethical committee, and written consent was obtained after explaining in detail the entire research protocol. Inclusion criteria for the present study included:

- Patients aged between 15 and 25 years

- Patients who underwent the procedure of canine retraction as a part of their fixed orthodontic treatment

- Patients without history of any systemic illness

- Patients with absence of any known inflammatory condition or diseases

- Patients without any known drug allergy

- Patients with good oral hygiene status

- Nonsmokers

- Patients with healthy periodontal status as evident from radiographic and clinical examination

- Patients with negative history of anti-inflammatory drug use in the past 2 months.

Armamentarium used in the present study included:

- Volumetric microcapillary pipette

- Polypropylene tubes

- Phosphate-buffered saline (PBS)

- Enzyme-linked immunosorbent assay (ELISA) kit for the analysis of PTX-3 levels.

Preadjusted edge-wise appliances were used in the selected patients. Upper arches were aligned and leveled followed by starting of canine retraction procedures. Active tieback and NiTi coil spring were used on the maxillary right and left quadrant respectively. Dontrix gauge was used for the measurement of the applied orthodontic forces. Collection of the gingival crevicular fluid (GCF) samples was done 1 hour prior to the start of the orthodontic canine retraction treatment. Collection of the GCF samples was done at following time intervals: 1 hour, 1, 7, and 14 days after the start of canine retraction procedure. Collection of the GCF samples was done from the distal side of the maxillary canines. Isolation of the sites was done with cotton and saliva ejector devices with the purpose of avoiding salivary contamination. Calibrated microcapillary pipettes were used for obtaining the GCF samples. Collection of approximately $5 \mu \mathrm{L}$ of the GCF samples was done in all the cases. Elution of the absorbed GCF was done in PBS solution maintaining the $\mathrm{pH}$ of 7.2. Polypropylene tubes were used for storing the eluted samples at $20^{\circ} \mathrm{C}$ prior to analysis. The ELISA kit was used for analysis of the samples as per manufacturer's instructions. All the data were recorded and compiled. All the results were analyzed by Statistical Package for the Social Sciences software version 16.0. Chi-square test, Student's t-test, and one-way analysis of variance were used for assessment of level of significance; $\mathrm{p}<0.05$ was taken as significant.

\section{RESULTS}

The value of PTX-3 in the GCF in the two study groups at different time intervals is shown in Table 1 and Graph 1. At the initial baseline time, the mean PTX-3 values in the GCF were found to be 1.32 and $1.34 \mathrm{ng} / \mathrm{mL}$ in the active tieback group and the NiTi coil spring group respectively. At 1-hour time period, the mean time intervals were found to be 1.70 and $1.73 \mathrm{ng} / \mathrm{mL}$ in the active tieback group and the NiTi coil spring group respectively. After

Table 1: Correlation of levels of PTX-3 in the GCF in two study groups

\begin{tabular}{llll}
\hline & \multicolumn{2}{c}{ Mean PTX-3 values $(\mathrm{ng} / \mathrm{mL})$} & \\
\cline { 2 - 3 } Time intervals & Active tieback & NiTi coil spring & p-value \\
\hline Initial baseline time & 1.32 & 1.34 & 0.65 \\
1 hour & 1.70 & 1.73 & 0.28 \\
24 hours & 2.71 & 2.82 & $0.02^{*}$ \\
1 week & 1.91 & 2.61 & $0.01^{*}$ \\
2 weeks & 1.45 & 2.15 & $0.02^{*}$ \\
\hline
\end{tabular}

*Significant

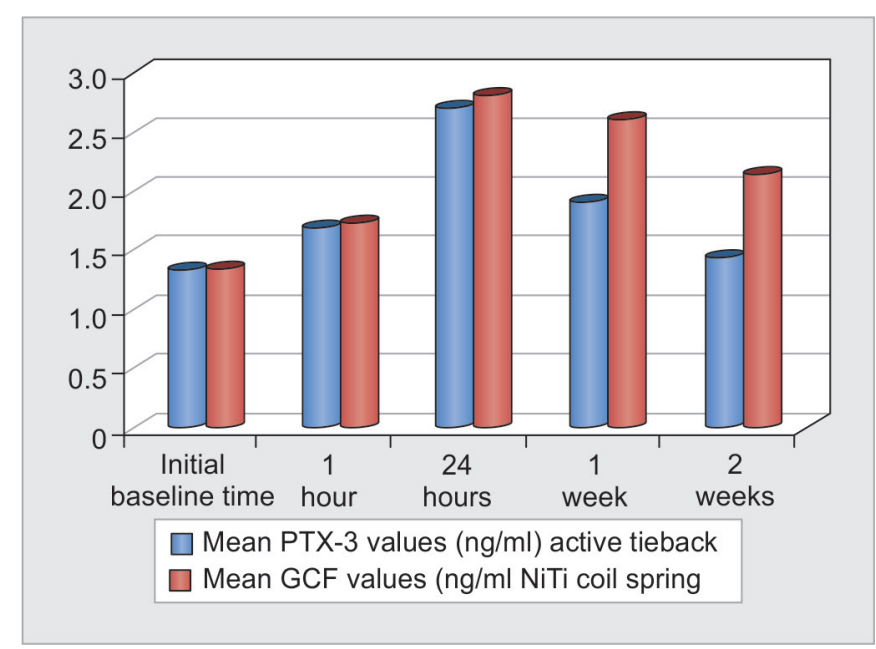

Graph 1: Mean PTX-3 and GCF values at different time intervals 
Table 2: Comparative evaluation of PTX-3 levels in GCF at different time intervals

\begin{tabular}{lll}
\hline & \multicolumn{2}{c}{$p$-value } \\
\cline { 2 - 3 } Time intervals & $\begin{array}{l}\text { Active tieback } \\
\text { group }\end{array}$ & $\begin{array}{l}\text { NiTi coil } \\
\text { spring group }\end{array}$ \\
\hline Initial baseline time vs 1 hour & $0.02^{*}$ & $0.01^{*}$ \\
Initial baseline time vs 24 hours & $0.01^{*}$ & $0.02^{*}$ \\
Initial baseline time vs 1 week & $0.03^{*}$ & $0.02^{*}$ \\
Initial baseline time vs 2 weeks & $0.02^{*}$ & $0.01^{*}$ \\
1 hour vs 24 hours & $0.01^{*}$ & $0.03^{*}$ \\
1 hour vs 1 week & $0.02^{*}$ & $0.01^{*}$ \\
1 hour vs 2 weeks & $0.02^{*}$ & $0.01^{*}$ \\
24 hours vs 1 week & $0.01^{*}$ & $0.02^{*}$ \\
24 hours vs 2 weeks & $0.03^{*}$ & $0.02^{*}$ \\
1 week vs 2 weeks & $0.01^{*}$ & $0.03^{*}$ \\
\hline
\end{tabular}

*Significant

24 hours, the mean value of PTX-3 in the active tieback group and NiTi coil spring group was found to be 2.71 and $2.81 \mathrm{ng} / \mathrm{mL}$ respectively. We observed a nonsignificant difference in the values of PTX-3 at initial baseline time and 1 hour. However, at 1 day, 1 , and 2 weeks time, we observed statistically significant difference in the mean values of PTX-3 between the two study groups $(\mathrm{p}<0.05)$. Table 2 highlights the comparative evaluation of PTX-3 levels in GCF at different time intervals. Significant difference was observed while comparing the mean PTX-3 values in between different time intervals in both the study groups.

\section{DISCUSSION}

The GCF reflects various events occurring in the periodontium and, therefore, can be used to assess levels of different biomarkers. ${ }^{8}$ Expression of various biologically active substances in the GCF in various periodontal diseases has been highlighted and well documented in the literature. Earlier studies have tried to assess the levels of PTX-3 in GCF in healthy periodontal tissue and during orthodontic treatment. ${ }^{5,9,10}$ Hence, we undertook the present study to assess and compare the level of PTX-3 in patients undergoing canine retraction with active tieback and NiTi coil spring.

In the present study, we observed an increase in the PTX-3 levels with time reaching to the peak levels at 24 hours time (Table 1). After this increase, we observed a fall in its value at the end of 7 and 14 days time period in both the study groups (Graph 1). Significant difference was obtained while comparing the mean PTX-3 values between different time intervals in both the study groups $(p<0.05)$ (Table 2). Our results were in correlation with the results obtained by Patel et al ${ }^{11}$ who reported similar findings in their study. Alfaqeeh and Anil ${ }^{12}$ assessed changes occurring in the levels of lactate dehydrogenase (LDH) in the GCF in patients undergoing active orthodontic treatment. They analyzed 20 patients that required first molar extraction as a part of orthodontic treatment and were treated with conventional therapy comprising straight wire mechanotherapy. They used $125 \mathrm{gm}$ of nitinol closed coil springs for carrying out the procedure of canine retraction. Contralateral canine teeth were taking as controls in their study. Before the initiation of the canine retraction process, they collected GCF from the canines followed by collection at 1 hour, 1 day, 1, 2, and 3 weeks. They compared the GCF LDH values with the control sites. They observed significantly higher LDH values at 1, 2, and 3 weeks respectively. They also observed significant increase in the levels from the baseline estimation time to the 3 weeks' time. From their results, the authors concluded that CGF can be used for the successful estimation of the LDH and active tooth movement can be actively indicated by its increasing levels. The PTX-3 levels were measured by Surlin et $\mathrm{al}^{10}$ in the GCF of the patients undergoing orthodontic treatment for assessment of correlation with various inflammatory markers. Paper strips were used for collection of GCF samples from 16 young patients and 13 adult patients undergoing active orthodontic treatment involving distalization of maxillary canine. They observed a significant increase in the PTX-3 GCF levels reaching maximum values at the time of 24 hours when compared with baseline values. From the results, they suggested that in periodontal orthodontic remodeling, PTX-3 is actively involved. Levels of PTX-3 were measured in the GCF of the patients undergoing canine retraction by active tieback and NiTi coil spring by Patel et al. ${ }^{11}$ They analyzed 15 patients that underwent first premolar extraction and later underwent canine retraction. In the same patient, 1 month after the placement of stainless steel wire, they started canine retraction by active tieback and NiTi coil spring in upper left and right quadrant respectively. One hour before the placement and commencement of the canine retraction, they measured the PTX-3 levels in the GCF followed by its measurement at 1 hour, 1 day, 1 , and 2 weeks respectively. They collected the GCF and assessed the PTX-3 levels. They observed statistically significant difference in the PTX-3 levels in the CGF of the patients undergoing canine retraction by active tieback and NiTi coil spring.

In patients who underwent periodontal surgery for the treatment of gingival overgrowth, Rauten et $\mathrm{al}^{13}$ assessed the PTX-3 and thrombospondin 1 levels during orthodontic treatment by assessment of their levels in GCF. They analyzed a total of 19 patients who presented with gingival overgrowth as a consequence of fixed orthodontic treatment. Gingivectomy procedure was used for the removal of the gingival overgrowth. From one half of the mandibular arch, the gingival overgrowth tissue 
was removed with magnification, while from the other half without magnification respectively. The ELISA tests were used for the estimation of PTX-3 levels and thrombospondin 1 levels. At different time intervals, the authors observed statistically significant difference between the levels of the two biomarkers. From the results, the authors concluded that a reduction in the process of inflammation might be suggested by the dynamic of PTX-3 and thrombospondin 1 levels.

\section{CONCLUSION}

From the above results, the authors concluded that in patients undergoing orthodontic treatment, PTX-3 is associated with periodontal remodeling under the effect of orthodontic forces. Also, in comparison to the active tieback, NiTi coil exhibited faster space closure rate.

\section{REFERENCES}

1. Vaden, JL.; Dale, JG.; Klontz, HA. The tweed-merrifield edgewise appliance: Philosophy, diagnosis, and treatment. In: Graber TM, Vanarsdall RL, editors. Orthodontics: current principle sand techniques. 4th ed. St. Louis (MO): Mosby; 2005. p. 675-715.

2. Hans MG, Groisser G, Damon C, Amberman D, Nelson S, Palomo JM. Cephalometric changes in overbite and vertical facial height after removal of 4 first molars or first premolars. Am J Orthod Dentofacial Orthop 2006 Aug;130(2):183-188.

3. Jan HU. Restore a wide radiant smile without dental extractions. Pak Oral Dent J 2005 Jun;25(1):65-68.

4. Natt AS, Sekhon AK, Munjal S, Duggal R, Holla A, Gupta P, Gandhi P, Sarin S. A comparative evaluation of static frictional resistance using various methods of ligation at different time intervals: an in vitro study. Int J Dent 2015 Apr;2015:1-7.

5. Pradeep AR, Kathariya R, Raghavendra NM, Sharma A. Levels of pentraxin-3 in gingival crevicular fluid and plasma in periodontal health and disease. J Periodontol 2011 May;82(5): 734-741.

6. McLaughlin, RP.; Bennet, JC.; Trevisi, H. Systemised orthodontic treatment mechanics. 1st ed. London: Mosby; 2001. p. 275-277.

7. Watanabe $\mathrm{Y}$, Miyamoto K. A nickel titanium canine retraction spring. J Clin Orthod 2002 Jul;36(7):384-388.

8. Goodman AR, Levy DE, Reis LF, Vilcek J. Differential regulation of TSG-14 expression in murine fibroblasts and peritoneal macrophages. J Leukoc Biol 2000 Mar;67(3):387-395.

9. Jaillon S, Peri G, Delneste Y, Frémaux I, Doni A, Moalli F, Garlanda C, Romani L, Gascan H, Bellocchio S, et al. The humoral pattern recognition receptor PTX3 is stored in neutrophil granules and localizes in extracellular traps. J Exp Med 2007 Apr;204(4):793-804.

10. Surlin P, Rauten AM, Silosi I, Foia L. Pentraxin-3 levels in gingival crevicular fluid during orthodontic tooth movement in young and adult patients. Angle Orthod 2012 Sep;82(5): 833-838.

11. Patel P, Shanthraj R, Bhagyalakshmi A, Garg N, Vallakati A. Comparative evaluation of pentraxin 3 levels in GCF during canine retraction with active tieback and NiTi coil spring: an in vivo study. J Orthod Sci 2016 Apr-Jun;5(2):52-56.

12. Alfaqeeh SA, Anil S. Lactate dehydrogenase activity in gingival crevicular fluid as a marker in orthodontic tooth movement. Open Dent J 2011 Jul;5:105-109.

13. Rauten AM, Silosi I, Stratul SI, Foia L, Camen A, Toma V, Cioloca D, Surlin V, Surlin P, Bogdan M. Expression of pentraxin 3 and thrombospondin 1 in gingival crevicular fluid during wound healing after gingivectomy in postorthodontic patients. J Immunol Res 2016 Jun;2016(1):1-7. 